\title{
Toxicidade ocular causada pelo tamoxifeno: relato de caso
}

\author{
Ocular toxicity caused by tamoxifen:case report
}

\author{
Eliane Terumi Inada ${ }^{1}$ \\ Karina Paula Watanabe ${ }^{2}$ \\ Sérgio Gustavo Tanaka ${ }^{3}$ \\ Luís Antônio Sakakisbara ${ }^{4}$
}

Trabalho realizado na disciplina de Oftalmologia do Hospital de Base da Faculdade de Medicina de São José do Rio Preto. São José do Rio Preto (SP).

${ }^{1}$ Residente de Oftalmologia da Faculdade de Medicina de São José do Rio. São José do Rio Preto (SP).

${ }^{2}$ Residente de Oftalmologia da Faculdade de Medicina de São José do Rio Preto. São José do Rio Preto (SP).

${ }^{3}$ Ex-residente de Oftalmologia da Faculdade de Medicina de São José do Rio. São José do Rio Preto (SP).

${ }^{4}$ Professor Assistente da Disciplina de Oftalmologia, Chefe do Setor de Retina da Faculdade de Medicina de São José do Rio. São José do Rio Preto (SP).

Endereço para correspondência: Eliane Terumi Inada - Av. Vera Cruz, 828/22 - São José do Rio Preto (SP) CEP 15085-010

E-mail: T_inada@ig.com.br

Recebido para publicação em 24.05.2004

Versão revisada recebida em 03.12.2004

Aprovação em 22.02.2005

\section{RESUMO}

O objetivo desse trabalho é relatar um caso de toxicidade ocular pelo tamoxifeno. Para isso, aferiu-se a melhor acuidade visual corrigida de ambos os olhos em tabela de Snellen. Foram realizados biomicroscopia do segmento anterior, refração, oftalmoscopia, angiofluoresceinografia e retinografia numa paciente de 63 anos, sexo feminino, cor branca, em uso de tamoxifeno $20 \mathrm{mg} /$ dia há 4 anos, com acuidade visual corrigida de 20/70 e 20/40. A biomicroscopia do segmento anterior apresentava ceratopatia verticilata e catarata nuclear e cortical posterior de 1+/4 em ambos os olhos. À oftalmoscopia, foi verificado alteração do brilho macular de ambos os olhos. E a angiofluoresceinografia mostrou hiperfluorescência na área macularem fase precoce (defeito em janela). Relata-se um caso de ceratopatia e maculopatia causadas pelo tamoxifeno.

Descritores: Neoplasias mamárias/quimioterapia; Tamoxifeno/administração \& dosagem; Tamoxifeno/efeitos adversos; Toxicidade de drogas; Degeneração macular; Fundo de olho; Mácula lútea; Angiofluoresceinografia; Doenças retinianas/induzido quimicamente; Relato de caso

\section{INTRODUÇÃO}

O tamoxifeno é uma droga antiestrogênica usada no tratamento do adenocarcinoma de mama, principalmente o tipo estrogênio dependente, e na terapia pós-operatória. Seus efeitos colaterais sistêmicos, na dose de $20 \mathrm{mg} /$ dia, incluem: cefaléia, náuseas, vômitos e alterações na contagem de células sangüíneas ${ }^{(1-2)}$.

Reações tóxicas oculares são relativamente raras, com uma incidência na literatura variando de 0,9 a $12 \%$, consistindo de retinopatia cristalina, depósitos corneanos, neurite óptica e edema macular cistóide ${ }^{(3-7)}$. O tempo de administração da droga que pode induzir a toxicidade ocular é variável, sendo que na maioria dos casos de complicações retinianas, estas ocorreram em pelo menos 1 ano após o início do tratamento ${ }^{(7)}$.

\section{OBJETIVO}

Relatar um caso de toxicidade ocular pelo tamoxifeno.

\section{MÉTODOS}

XXX, 63 anos, sexo feminino, cor branca, hipertensa, com história de câncer de mama, submetida a mastectomia radical à Patey à esquerda e a um subseqüente tratamento quimioterápico ( 7 ciclos de ciclofosfamida + methotrexate + fluoracil). Veio ao nosso serviço em uso de tamoxifeno $(20 \mathrm{mg} / \mathrm{dia})$ há 4 anos, Captopril (12,5 mg 8/8 horas), Hidroclorotiazida ( $25 \mathrm{mg} / \mathrm{dia})$ e 
Propranolol (40 mg 12/12 horas). Realizado exame oftalmológico constituído por: refração (tabela de Snellen), biomicroscopia do segmento anterior, oftalmoscopia, angiofluoresceinografia e retinografia de ambos os olhos (AO).

\section{RESULTADOS}

Constatou-se acuidade visual corrigida de 20/70 e 20/40, em olho direito e esquerdo, respectivamente, córnea transparente com opacidade subepitelial linear inferior ao eixo visual (ceratopatia verticilata) e catarata nuclear e cortical posterior
+/4+ em AO. À oftalmoscopia, foi verificado diminuição do brilho macular bilateral (Figura 1) e a angiofluoresceinografia mostrou hiperfluorescência da área macular em fase precoce em AO, que foi interpretada como defeitos em janela do epitélio pigmentar da retina causados por atrofia (Figura 2).

\section{DISCUSS ÃO}

A fisiopatogenia induzida pelo tamoxifeno permanece desconhecida $^{(7)}$. Engelke et al. sugere que a droga cause mudanças na composição lipídica do epitélio pigmentar da retina ${ }^{(8)}$.

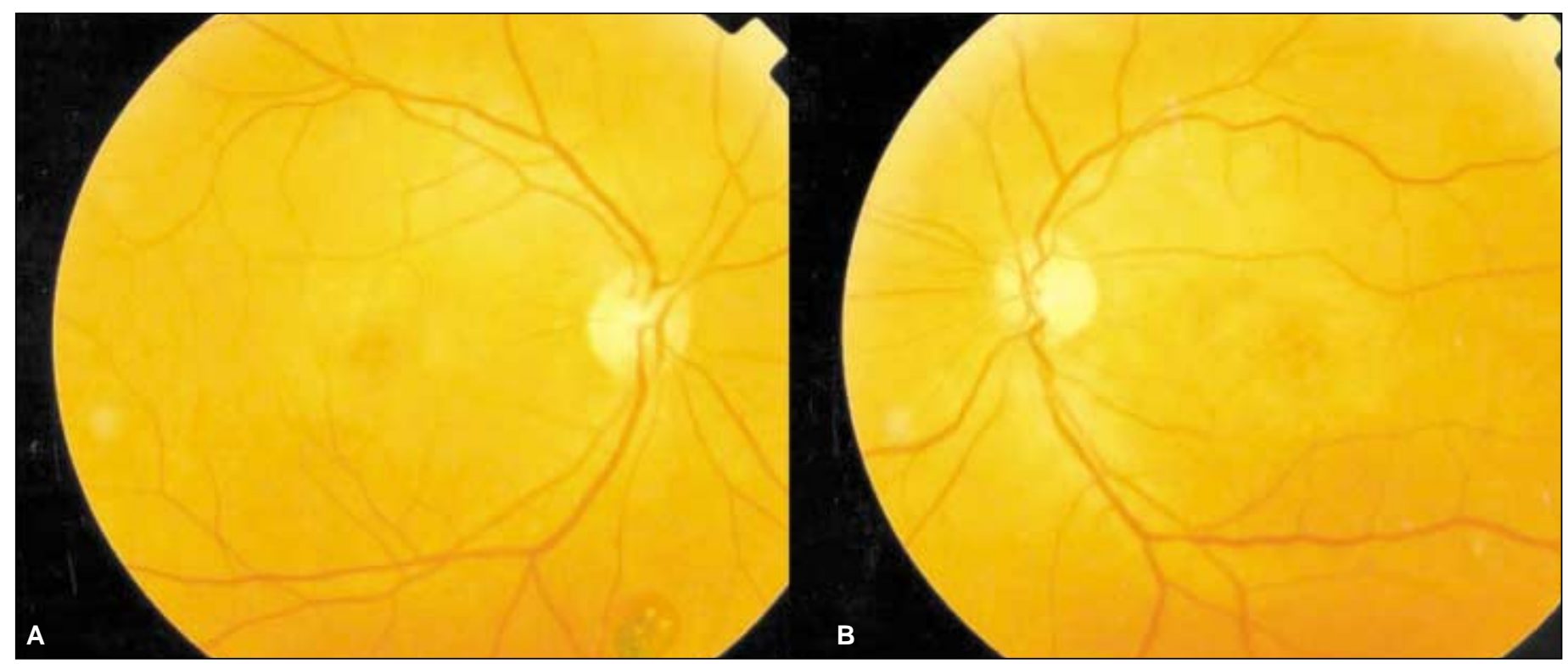

Figura 1 - Maculopatia por tamoxifeno. A: olho direito; B: olho esquerdo

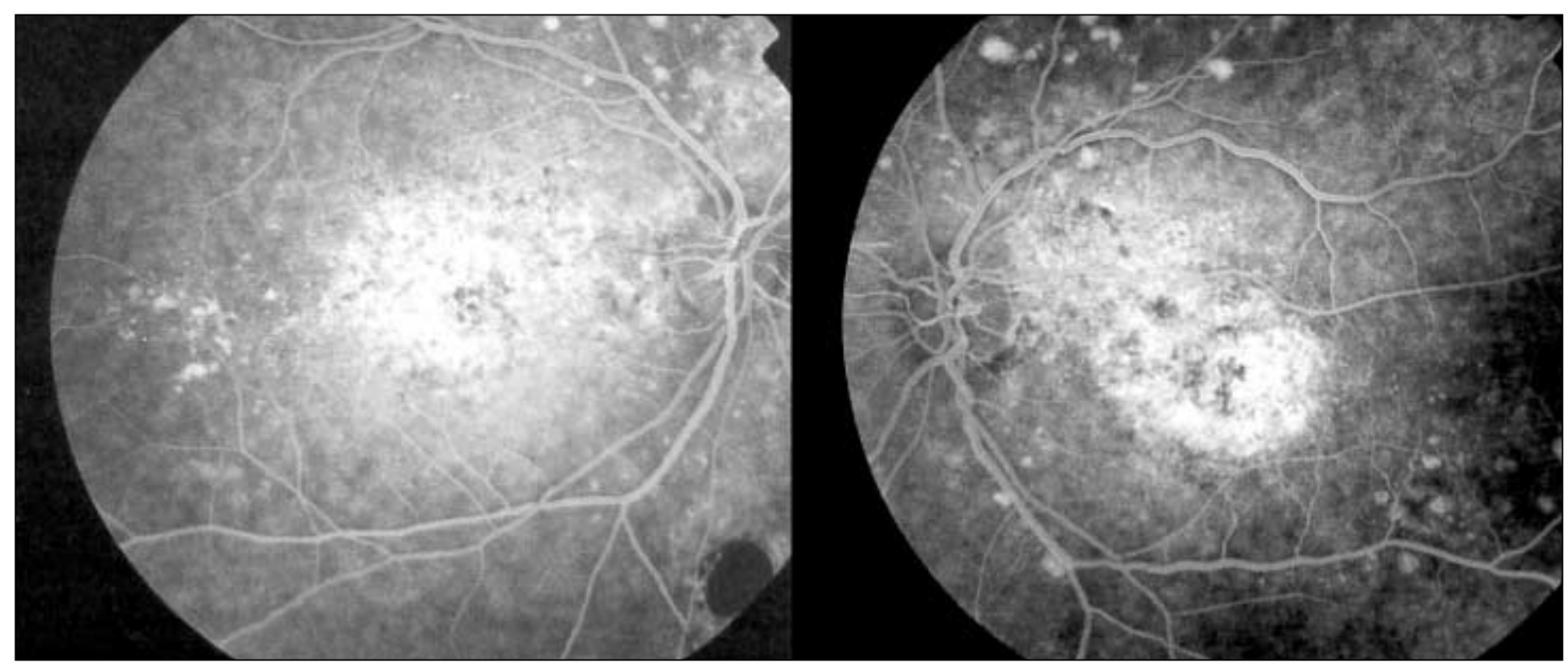

Figura 2 - Hiperfluorescência em área macular devido atrofia do epitélio pigmentar da retina 
A retinopatia pelo tamoxifeno foi inicialmente relatada em 4 pacientes que tinham recebido doses de 108 a $230 \mathrm{~g}$ de tamoxifeno durante um período de 17 a 27 meses $^{(9)}$. A toxicidade retiniana associada a baixas doses foi presenciada em 2 casos de 17 pacientes tratadas com $30 \mathrm{mg} / \mathrm{dia}^{(10)}$. Um estudo prospectivo de 63 pacientes tratadas com tamoxifeno, $20 \mathrm{mg} / \mathrm{dia}$, demonstrou retinopatia em 4 pacientes $^{(7)}$. O eletrorretinograma multifocal em 15 pacientes em tratamento com a mesma dosagem acima, não demonstrou efeitos retino-tóxicos ${ }^{(11)}$.

A retinotoxicidade ocorre na forma de depósitos anelares bilaterais, múltiplos, amarelados, cristalinos localizados na mácula. As lesões, se severas, podem causar diminuição de acuidade visual ${ }^{(12)}$. Outros efeitos colaterais oculares incluem ceratopatia verticilata e raramente neuropatia óptica bilateral reversível em caso de suspensão do tratamento ${ }^{(7,9-10,13)}$.

O tamoxifeno é um agente hormonal bem tolerado sem graves efeitos colaterais. Retinopatia e ceratopatia têm sido relatadas em pacientes tratados principalmente com altas doses da droga e por longo período de tempo ${ }^{(7)}$. Uma triagem de rotina para detecção de complicações oculares parece ser desnecessária; entretanto, um índice de suspeita deve ser mantido. Cessar o tratamento usualmente previne uma deterioração adicional mas não resulta em recuperação visual.

\section{ABSTRACT}

To report tamoxifen ocular toxicity. The best visual acuity was measured in both eyes with Snellen chart, slit-lamp examination of anterior segment, refraction, dilated fundus examination, fluorescein angiography and retinography in a 63-yearold patient, female, white, using tamoxifen $20 \mathrm{mg} /$ day for 4 years, with 20/70 and 20/40 corrected visual acuity. The anterior segment examination showed corneal linear subepithelial opacity inferior to the visual axis and nuclear and posterior cortical cataract $(1+/ 4)$ in both eyes. Fundus examination showed alteration of macular color in both eyes. Fluorescein angiography presented hyperfluorescence in the macular area at an early phase (window defect). Report of keratopathy and maculopathy caused by tamoxifen.

Keywords: Breast neoplasms/drug therapy; Tamoxifen/administration \& dosage; Tamoxifen/adverse effects; Drug toxicity; Macular degeneration; Fundus oculi; Macula lutea; Fluorescein angiography; Retinal diseases/chemically induced; Case report.

\section{REFERÊNCIAS}

1. Imperia PS, Lazarus HM, Lass JH. Ocular complications of systemic cancer chemotherapy. Surv Ophthalmol. 1989;34(3):209-30.

2. Sananes S, Khairallah T, Touboul E, Salat - Baroux J, Uzan S. [A surveillance of patients treated with tamoxifen]. Presse Med. 1996;25(10):499-502. French.

3. Noureddin BN, Seoud M, Bashshur Z, Salem Z, Shamseddin A, Khalil A. Ocular Toxicity in low - dose tamoxifen: a prospective study. Eye. 1999;13(Pt 6):729-33.

4. Lazzaroni F, Scorolli L, Pizzoleo CF, Savini G, De Nigris A, Giosa F, et al. [Tamoxifen retinopathy: does it really exist?] Graefes Arch Clin Exp Ophthalmol. 1998;236(9):669-73. Germany.

5. Tang R, Shields J, Schiffman J, Li H, Locher D, Hampton J, et al. Retinal changes associated with tamoxifen treatment for breast cancer. Eye. 1997;11 (Pt 3):295-7. Comment in: Eye. 1998;12(Pt 3a):485-6.

6. Heier JS, Dragoo RA, Enzenauer RW, Waterhouse WJ. Screening for ocular toxicity in asymptomatic patients treated with tamoxifen. Am J Ophthalmol. 1994;117(6):772-5.

7. Pavilidis NA, Petris C, Briassoulis E, Klouvas G, Psilas C, Rempapis J, et al Clear evident that long-term, low-dose tamoxifen treatment can induce ocular toxicity: a prospective study of 63 patients. Cancer. 1992;69(12):2961-4.

8. Engelke M, TykHonova S, Zorn-Kruppa M, Diehl H. Tamoxifen induces changes in the lipid composition of the retinal pigment epithelium cell line D407. Pharmacol Toxicol. 2002;91(1):13-21.

9. Kaiser-Kupfer MI, Lippman ME. Tamoxifen retinopathy. Cancer Treat Rep. 1978;62(3):315-20.

10. Vinding T, Nielsen NV. Retinopathy caused by treatment with tamoxifen in low dosage. Acta Ophthalmol (Copenh). 1983;61(1):45-50.

11. Berezovsky A, Salomão SR, Pereira JM, Sacai PY, Yuri P, Motono M Eletrorretinograma multifocal em pacientes tratadas com tamoxifeno em baixa dosagem. Arq Bras Oftalmol. 2004;67(4):631-5.

12. Kanski JJ. Oftalmologia clínica: uma abordagem sistemática. 4a ed. São Paulo: Manole; 2004

13. Pugesgaard T, Von Eyben FE. Bilateral optic neuritis evolved during tamoxifen treatment. Cancer. 1986;58(2):383-6.

\section{ABO ELETRônICO}

\section{Acesso: http://www.abonet.com.br}

